\title{
Getting to the point in point-of-care diagnostics for tuberculosis
}

\author{
Elisa H. Ignatius, ${ }^{1,2}$ Keira A. Cohen, ${ }^{3}$ and William R. Bishai' \\ 'Division of Infectious Diseases, ${ }^{2}$ Division of Clinical Pharmacology, and ${ }^{3}$ Division of Pulmonary and Critical Care Medicine, Johns Hopkins University School of Medicine, Baltimore, Maryland, USA.
}

\begin{abstract}
Tuberculosis (TB) continues to affect over 10 million people per year worldwide. Despite advances in diagnosis, smear microscopy insufficiently detects pulmonary disease, with test result reporting taking longer than a day. While urine assays to detect the lipopolysaccharide lipoarabinomannan (LAM), present in mycobacterial cell walls, can provide results within minutes, the currently available assay has low sensitivity and its application is limited to patients with HIV suspected of having TB. In this issue of the $J C I$, Broger and Nicol et al. investigated 3 rapid urine tests in 372 ambulatory HIV-negative individuals suspected of having TB in South Africa and Peru. FujiLAM emerged as a rapid test to confirm TB diagnosis in the HIVseronegative population. This study shows that FujiLAM has considerable potential to reshape the TB diagnostics landscape, making diagnosis and treatment in one office visit a reality for TB.
\end{abstract}

\section{Rapid tests for pulmonary TB}

Despite the World Health Organization's (WHO) designation of tuberculosis (TB) as a global health emergency nearly 30 years ago, a true point-of-care diagnostic test for TB disease remains elusive. Since its discovery by German physician Robert Koch in the late 1800 s, sputum smear microscopy - the initial rapid test for pulmonary TB - has undergone various adaptations to increase the sensitivity of detection. Even with these advances, smear microscopy detects only $55 \%-86 \%$ of culture-positive pulmonary TB cases (1), and reporting of test results is not usually available the same day. When Xpert MTB/RIF, a cartridge-based molecular assay, launched in 2010, it was considered a game changer for TB diagnostics due to increased sensitivity for smear-negative disease (2-4) and the promise of test results within 2 hours. However, global rollout of Xpert MTB/
RIF has been predominantly in public sector reference labs where delays in sample transport and data return often prevent same-day reporting of test results (5-8). Therefore, true point-of-care testing available in minutes for the diagnosis of active TB disease remains a high priority.

Lipoarabinomannan (LAM) is a lipopolysaccharide present in mycobacterial cell walls, and assays to detect its presence in urine have been proposed as a noninvasive, point-of-care, biomarker-based test-much akin to the commonly used urine antigen tests for Streptococcus pneumoniae and Histoplasma capsulatum. The currently available assay, AlereLAM, which takes 50 to 60 minutes to perform, has a low sensitivity $(14 \%-18 \%)$, but reasonable specificity ( $91 \%-95 \%)$ in immunocompetent patients (9-11). However, in patients with HIV, the sensitivity rises to $42 \%-56 \%$ and the specificity remains high

Related Article: p. 5756

Conflict of interest: KAC has received consulting fees from Insmed, Hillrom, and Merck. WRB is a shareholder in Essa Pharma Inc. and Viking Therapeutics Inc., and is an inventor on a TB diagnostics patent, US20150104819A1, "Methods of using isoniazid for the diagnosis of lung infections." Copyright: @ 2020, American Society for Clinical Investigation. Reference information: J Clin Invest. 2020;130(11):5671-5673. https://doi.org/10.1172/JCI142497.

at $91 \%-94 \%(11,12)$, with positive predictive values (PPVs) and negative predictive values (NPVs) at approximately $84 \%$ and $57 \%$, respectively (9). Thus, when positive, AlereLAM can rule in a high likelihood of TB in a person with HIV, but it has little utility when it is negative.

Based on the above urine LAM performance data, coupled with reduced mortality among patients with HIV who screen positive and are promptly initiated on TB treatment (13), the 2015 WHO guidelines recommend use of AlereLAM for evaluation of patients with HIV suspected of having TB. Despite this WHO recommendation, a recent study found that uptake of AlereLAM was limited to only $21 \%$ of high-burden TB countries. Respondents cited budget constraints, limited countryspecific data, administrative barriers, insufficient coordination between TB and HIV programs, and perceived small eligible population as primary limitations (14). The recommendation to use urine LAM assays only in patients with HIV substantially limits the test's utility, as HIV-negative patients with pulmonary TB account for over $90 \%$ of the global TB burden (15).

\section{Enabling rapid TB treatment initiation}

In this issue of the JCI, Broger and Nicol et al. investigated a recently developed and more accurate urine LAM test (FujiLAM) for its sensitivity and specificity in active pulmonary TB. Importantly, the researchers restricted their analysis to ambulatory patients without HIV suspected of having TB in South Africa and Peru (16). In their population of 372 individuals with suspected pulmonary TB, the FujiLAM test demonstrated 53\% sensitivity and 99\% specificity when using sputum mycobacterial culture as the gold standard. They also noted that in a setting with TB prevalence of 30\%, FujiLAM has a PPV of $95.2 \%$, an NPV of $83.2 \%$, and a positive likelihood ratio of 46.2 (16).

Broger and Nicol et al. also tested an experimental, laboratory-based assay, Ecl- 
LAM, which requires centrifugation and electrochemiluminescence. EclLAM was observed to have an even greater sensitivity than FujiLAM (66.7\%), supporting the notion that most patients with pulmonary TB have detectable urinary LAM, albeit in low concentrations, and offering hope that even more sensitive, next-generation urine LAM tests may emerge. Overall, the authors concluded that FujiLAM's sensitivity for identifying active TB in individuals without HIV suspected of having TB is 5 times that of the current commercially available test, AlereLAM. The authors rightly point out that this improved sensitivity makes FujiLAM a candidate to serve as a 1-hour rule-in test for TB among individuals without HIV, which may in turn enable rapid TB treatment initiation within a single clinic visit (16).

\section{Conclusions and considerations}

There are some important limitations to this study (16). First, using frozen rather than prospectively collected fresh urine samples in this investigation deviates from how this assay would be applied clinically. However, the freezing process appears to reduce sensitivity slightly (17), therefore, the observed results may actually underestimate real-world performance. Specimen centrifugation may have also contributed to increased detection. These analytic comparisons should be reproduced using fresh, noncentrifuged urine in order to more definitively demonstrate the relative sensitivity and specificity of FujiLAM compared with other LAM assays. FujiLAM was observed to have a higher sensitivity in Peru (64.6\%) than in South Africa (25.0\%) (16). While it is possible that bacterial differences in LAM abundance could contribute to discrepant sensitivity of this assay in distinct geographic settings, a more likely explanation is that the baseline bacterial burden (bacillary load) between the study participants in each country cohort differed. The sensitivity of FujiLAM appears to correlate well with bacillary load, given that participant samples from Peru had a shorter time to positivity (TTP) on mycobacterial growth indicator tube (MGIT) and greater proportion smear and Xpert positive (16). Coupling FujiLAM with other diagnostics such as Xpert may compensate for imbalances such as bacillary load, and bring overall case identification to acceptable thresholds in both contexts.

While FujiLAM has previously been demonstrated to have high sensitivity (70.7\%) among patients with HIV, regardless of CD4 count (18), the observation of $53 \%$ sensitivity in subjects without HIV is a substantial improvement in the current urine LAM test (16). If the FujiLAM assay becomes commercially available, it will be important to consider whether and how this improved urine LAM should be integrated with the current TB diagnostic algorithms, including GeneXpert. In addition to the fact that FujiLAM detected $23.3 \%$ patients who were smear negative, it also detected $31.0 \%$ of patients who were positive on FujiLAM and negative on GeneXpert. Unfortunately, FujiLAM also misses $27.9 \%$ of smear-positive samples and $39.0 \%$ of Xpert-positive samples. Overall, the addition of FujiLAM to Xpert increases sensitivity from $76.6 \%$ to $82.0 \%$, with combined PPV and NPV of $96.8 \%$ and $92.8 \%$, respectively. These observations suggest that FujiLAM, used in conjunction with another standard microbiologic or molecular test, might further enhance diagnostic sensitivity. However, it is important to recognize that urine LAM tests do not provide insight into mycobacterial drug susceptibility patterns, thus additional microbiologic testing may be required in areas with a high prevalence of drug-resistant TB. Future applications of FujiLAM might include identification of high-risk patients on the precipice of progression from latent infection to active TB disease (19), longitudinal monitoring of patients for treatment response (20), and evaluation in children. Extrapulmonary TB introduces even more challenges, as diagnosis may require invasive sampling, and both microbiologic as well as molecular diagnostic tests have much lower sensitivity in these cases (21). Whether or not urine LAM has a role in low-burden TB settings remains to be explored. One potential consideration in these settings is cross-reactivity with nontuberculous mycobacterial species $(22,23)$.

Imperfect diagnostic tools contribute to ongoing challenges in TB control. Broger and Nicol et al. show that FujiLAM has considerable potential to reshape the TB diagnostics landscape and make true same-day-visit diagnosis and treatment a reality for TB. With the COVID-19 pandemic threatening to disrupt recent gains in TB control, even modest improvements in $\mathrm{TB}$ treatment initiation rates may prove valuable $(24,25)$. Based on these data and additional supportive field testing, FujiLAM may well be incorporated into future guidelines for individuals without HIV suspected of having TB.

\section{Acknowledgments}

EHI is supported by T32 GM06669117. KAC reports research support funding from the Burroughs Wellcome Fund Career Award for Medical Scientists, the Cystic Fibrosis Foundation, and NIH grant K08 HL139994. WRB gratefully acknowledges the support of NIH grant AI 037856.

Address correspondence to: William R. Bishai, 1550 Orleans Street, CRB2 Room 108, Baltimore, Maryland 21287, USA. Phone: 410.955.3507; Email: wbishai@ jhmi.edu.

1. Davis JL, Cattamanchi A, Cuevas LE, Hopewell PC, Steingart KR. Diagnostic accuracy of same-day microscopy versus standard microscopy for pulmonary tuberculosis: a systematic review and meta-analysis. Lancet Infect Dis. 2013;13(2):147-154.

2. Boehme CC, et al. Rapid molecular detection of tuberculosis and rifampin resistance. $N$ Engl J Med. 2010;363(11):1005-1015.

3. Small PM, Pai M. Tuberculosis diagnosis-time for a game change. $\mathrm{N} \mathrm{Engl} \mathrm{JMed.}$ 2010;363(11):1070-1071.

4. Berhanu RH, et al. Performance of Xpert MTB/ RIF, Xpert Ultra, and Abbott RealTime MTB for diagnosis of pulmonary tuberculosis in a high-HIV-burden setting. J Clin Microbiol. 2018;56(12):e00560-18.

5. Goh KS, Rastogi N. Rapid preliminary differentiation of species within the Mycobacterium tuberculosis complex: proposition of a radiometric method. Res Microbiol. 1991;142(6):659-665.

6. Albert H, Nathavitharana RR, Isaacs C, Pai M, Denkinger CM, Boehme CC. Development, rollout and impact of Xpert MTB/RIF for tuberculosis: what lessons have we learnt and how can we do better? Eur Respir J. 2016;48(2):516-525.

7. Churchyard GJ, et al. Xpert MTB/RIF versus sputum microscopy as the initial diagnostic test for tuberculosis: a cluster-randomised trial embedded in South African roll-out of Xpert MTB/RIF. Lancet Glob Health. 2015;3(8):e450-e457.

8. Kwak N, et al. Diagnostic accuracy and turnaround time of the Xpert MTB/RIF assay in routine clinical practice. PLoS One. 2013;8(10):e77456.

9. Mutetwa R, et al. Diagnostic accuracy of commercial urinary lipoarabinomannan detection in African tuberculosis suspects and patients. Int $J$ 
Tuberc Lung Dis. 2009;13(10):1253-1259.

10. Kroidl I, et al. Performance of urine lipoarabinomannan assays for paediatric tuberculosis in Tanzania. Eur Respir J. 2015;46(3):761-770.

11. Minion J, et al. Diagnosing tuberculosis with urine lipoarabinomannan: systematic review and meta-analysis. Eur Respir J. 2011;38(6):1398-1405.

12. Bjerrum S, et al. Lateral flow urine lipoarabinomannan assay for detecting active tuberculosis in people living with HIV. Cochrane Database Syst Rev. 2019;10:CD011420.

13. Peter JG, et al. Effect on mortality of pointof-care, urine-based lipoarabinomannan testing to guide tuberculosis treatment initiation in HIV-positive hospital inpatients: a pragmatic, parallel-group, multicountry, open-label, randomised controlled trial. Lancet. 2016;387(10024):1187-1197.

14. Singhroy DN, et al. Adoption and uptake of the lateral flow urine LAM test in countries with high tuberculosis and HIV/AIDS burden: current landscape and barriers. Gates Open Res. 2020;4:24 .

15. WHO. Global Tuberculosis Report 2019. https:// www.who.int/tb/publications/global_report/en.
Accessed August 31, 2020.

16. Broger T, et al. Diagnostic accuracy of three urine lipoarabinomannan tuberculosis assays in HIV-negative outpatients. J Clin Invest. 2020;130(11):5756-5764.

17. Broger T, Muyoyeta M, Kerkhoff AD, Denkinger $\mathrm{CM}$, Moreau E. Tuberculosis test results using fresh versus biobanked urine samples with FujiLAM. Lancet Infect Dis. 2020;20(1):22-23.

18. Broger T, et al. Diagnostic accuracy of a novel tuberculosis point-of-care urine lipoarabinomannan assay for people living with HIV: A meta-analysis of individual in- and outpatient data. PLoS Med. 2020;17(5):e1003113.

19. Correia-Neves M, et al. Biomarkers for tuberculosis: the case for lipoarabinomannan. ERJOpen Res. 2019;5(1):00115-2018.

20. Drain PK, Gounder L, Grobler A, Sahid F, Bassett IV, Moosa MY. Urine lipoarabinomannan to monitor antituberculosis therapy response and predict mortality in an HIV-endemic region: a prospective cohort study. BMJOpen. 2015;5(4):e006833.

21. Scott LE, et al. Diagnostic accuracy of Xpert MTB/RIF for extrapulmonary tuberculosis specimens: establishing a laboratory testing algorithm for South Africa.J Clin Microbiol. 2014;52(6):1818-1823.

22. Qvist $T$, et al. Urine lipoarabinomannan pointof-care testing in patients affected by pulmonary nontuberculous mycobacteria--experiences from the Danish Cystic Fibrosis cohort study. BMC Infect Dis. 2014;14:655.

23. Khoo KH, Tang JB, Chatterjee D. Variation in mannose-capped terminal arabinan motifs of lipoarabinomannans from clinical isolates of Mycobacterium tuberculosis and Mycobacterium avium complex. J Biol Chem. 2001;276(6):3863-3871.

24. Togun T, Kampmann B, Stoker NG, Lipman M. Anticipating the impact of the COVID-19 pandemic on TB patients and TB control programmes. Ann Clin Microbiol Antimicrob. 2020;19(1):21.

25. Stop TB partnership. The potential impact of the Covid-19 response on tuberculosis in highburden countries: a modelling analysis. http:// www.stoptb.org/assets/documents/news/ Modeling\%20Report_1\%20May\%202020_ FINAL.pdf. Accessed August 31, 2020. 